\title{
Alleviation of Anxiety/Depressive-Like Behaviors and Improvement of Cognitive Functions by Lactobacillus plantarum WLPL04 in Chronically Stressed Mice
}

\author{
Xuan Sun $\mathbb{D}^{1,2}$ Hong-Fei Zhang $\mathbb{D}^{1},{ }^{1}$ Chao-Lin Ma $\mathbb{D},{ }^{2}$ Hua Wei $\mathbb{D}^{3},{ }^{3}$ Bao-Ming Li $\mathbb{D}^{2}{ }^{2}$ \\ and Jie Luo $\mathbb{B D}^{4}$ \\ ${ }^{1}$ School of Life Sciences, Nanchang University, Nanchang 330031, China \\ ${ }^{2}$ Institute of Life Science, Nanchang University, Nanchang 330031, China \\ ${ }^{3}$ State Key Laboratory of Food Science and Technology, Nanchang University, Nanchang 330029, China \\ ${ }^{4}$ School of Public Health and Key Laboratory of Preventive Medicine, Nanchang University, Nanchang 330006, China
}

Correspondence should be addressed to Jie Luo; jieluo@ncu.edu.cn

Received 7 October 2020; Revised 18 November 2020; Accepted 17 January 2021; Published 31 January 2021

Academic Editor: Louis DeTolla

Copyright (c) 2021 Xuan Sun et al. This is an open access article distributed under the Creative Commons Attribution License, which permits unrestricted use, distribution, and reproduction in any medium, provided the original work is properly cited.

Background. Intestinal microorganisms play an important role in regulating the neurodevelopment and the brain functions of the host through the gut-brain axis. Lactobacillus, one of the most representative intestinal probiotics, produces important effects on human physiological functions. Our previous studies reveal that the Lactobacillus plantarum WLPL04 has a series of beneficial actions, such as antiadhesion of pathogens, protection from the harmful effect of sodium dodecyl sulfate, and anti-inflammatory stress on Caco2 cells. However, its effects on brain functions remain unknown. The present study aims to evaluate the potential effect of $L$. plantarum WLPL04 on anxiety/depressive-like behaviors in chronically restrained mice. Methods. Newly weaned mice were exposed to chronic restraint stress for four weeks and raised daily with or without L. plantarum WLPL04 water supplement. Animals were behaviorally assessed for anxiety/depression and cognitive functions. The $16 \mathrm{~S}$ rRNA sequencing was performed to analyze the intestinal microbiota structure. The levels of the medial prefrontal cortical (mPFC) brain-derived neurotrophic factor (BDNF)/tropomyosin-related kinase B (TrkB) and serum 5-hydroxytryptamine (5-HT) were examined using Western blot and enzyme-linked immunosorbent assay. Results. The chronic stress-induced anxiety/depressive-like behaviors and cognitive deficits were significantly alleviated by the $L$. plantarum WLPL04 treatment. The 16S rRNA sequencing analysis showed that the chronic stress reduced the diversity and the richness of intestinal microbiota, which were rescued by the L. plantarum WLPL04 treatment. The levels of BDNF and TrkB in the $\mathrm{mPFC}$ and the concentration of 5-HT in the serum remained unchanged in chronically restrained mice treated with the L. plantarum WLPL04. Conclusions. The L. plantarum WLPL04 can rescue anxiety/depressive-like behaviors and cognitive dysfunctions, reverse the abnormal change in intestinal microbiota, and alleviate the reduced levels of 5-HT, BDNF, and TrkB induced by chronic stress in mice, providing an experimental basis for the therapeutic application of $L$. plantarum on anxiety/depression.

\section{Introduction}

The chronic stress in early life increases the susceptibility to a range of psychopathologies, including depression and anxiety [1]. Traditional psychotropic medications are controversial partly because the long-term effects to developing nervous systems have not been clearly established [2]. Recently, accumulating evidence indicates the existence of a microbiome-gut-brain axis and strong bidirectional communications among these structures $[3,4]$. Stress in early life can alter the enteric microbiota [5], and intestinal bacterial infection can induce anxiety-like behaviors and cause memory deficits $[6,7]$. Thus, the regulation of the intestinal microbiota is suggested to be an interesting strategy for the development of new therapy for mental diseases [8].

Probiotics are a group of active microorganisms and confer health benefits to the host via active interactions with 
endogenous microbiota and gut cells when provided in appropriate amount [9]. Probiotics are also beneficial to patients suffering from psychiatric illness. In 2005, Logan and Katzman have used probiotics as adjunct therapy for depression for the first time [10]. Some strains of Lactobacillus and Bifidobacterium are shown to alleviate mood disorders and prevent stress-induced alterations in colonic microbiota $[8,11]$.

Lactic acid bacteria are regarded as safe and beneficial probiotics that may help prevent constipation, irritable bowel syndrome or Crohn's disease, and asthma or eczema in children $[12,13]$. A recent study shows that the Lactobacillus plantarum NDC75017 alleviates the learning and the memory deficit in aging rats by reducing the mitochondrial dysfunction [14]. The Lactobacillus plantarum MTCC1325 strain, which produces acetylcholine, has potential antioxidant and anti-Alzheimer activities against the D-galactose-induced Alzheimer's disease [15, 16]. Most L. plantarum strains are isolated from fermented food, whereas the strains isolated from human breastmilk have some special features, such as regulating natural and acquired immune responses, treating infectious mastitis, having antimicrobial properties, and having beneficial effects for infants [17-19]. A previous study shows that the $L$. plantarum WLPL04 isolated from human breastmilk can increase the capabilities of human body against pathogens and may be a candidate probiotic for promoting host health [20]. The present study aims to assess the effects of $L$. plantarum WLPL04 on anxiety/depressive-like behaviors and cognitive functional deficits induced by chronic stress in young adult mice and analyze the underlying mechanism.

\section{Materials and Methods}

2.1. Animals. A total of 30 male C57BL/6 mice (approximately four weeks old at the start of the experiments) were used. All mice were randomly assigned to three groups (10 mice per group), including one control group and two chronic restraint-stressed groups. One of the stressed groups was provided with normal drinking water, and the other stressed group was provided with normal drinking water and L. plantarum WLPL04, which was kindly provided by State Key Laboratory of Food Science and Technology, Nanchang University, China. The final concentration of the L. plantarum WLPL04 in drinking water was $10^{9} \mathrm{CFU} / \mathrm{mL}$. The mice were housed in cages ( 3 or 4 mice per cage) under a constant temperature $\left(23^{\circ} \mathrm{C}-25^{\circ} \mathrm{C}\right)$ and a $12 \mathrm{~h} \mathrm{light/dark}$ cycle with ad libitum access to food (SHOOBREE, SPF-grade chow, Jiangsu Xietong Pharmaceutical Bio-Engineering Co., Nanjing, Jiangsu, China) and water (sterilized drinking water). Mice were placed in a plastic restrainer in their home cages for $3 \mathrm{~h}$ daily (from 11 : 00 to 14:00) for 28 consecutive days to establish chronic restraint stress [21]. All experiments were carried out in accordance with the principles of laboratory animal care and use approved by the Nanchang University Animal Care and Use Committee Guidelines.
2.2. Behavioral Assessments. Prior to behavioral tests, all mice were handled for five days and received $1 \mathrm{~h}$ accommodation to the experiment room before testing. The open field, elevated plus maze, and forced swimming tests were performed to analyze anxiety/depressive-like behaviors, and the novel object recognition and Barnes maze tests were used to evaluate the cognitive functions of the medial prefrontal cortex (mPFC).

2.2.1. Open Field Test. The open field test was performed in accordance with previously described procedures [22]. Each mouse was placed in an open field arena $(45 \mathrm{~cm} \times 45 \mathrm{~cm} \times 40 \mathrm{~cm}$, Med Associates, Vermont, USA) and allowed to freely explore the arena for $10 \mathrm{~min}$ to carry out the test. The locomotion of the mouse was recorded using a video capture software. The total distance and the routine traveled in the arena were measured. The open field arena was cleaned with $75 \%$ ethanol after each use.

2.2.2. Elevated Plus Maze Test. The elevated plus maze test was performed in a gray plastic cross-shaped maze (Med Associates, Vermont, USA) with $1.0 \mathrm{~m}$ elevation from the floor, two open arms $(35 \mathrm{~cm} \times 7 \mathrm{~cm})$, and two closed arms $(35 \mathrm{~cm} \times 7 \mathrm{~cm} \times 40 \mathrm{~cm})$. The arms were connected by the center platform $(7 \mathrm{~cm} \times 7 \mathrm{~cm})$. A mouse was placed in the intersection of the open and closed arms, facing an open $\mathrm{arm}$, and allowed to freely explore the maze for $10 \mathrm{~min}$. The behavioral exploration and the time spent in the open and the closed arms were video recorded for analysis. The maze arms were cleaned with $75 \%$ ethanol after each use.

2.2.3. Forced Swimming Test. The forced swimming test was performed in a Plexiglas cylinder $(25 \mathrm{~cm}$ in height and $10 \mathrm{~cm}$ in diameter) containing water at height of $10 \mathrm{~cm}$ and temperature of $22^{\circ} \mathrm{C}-25^{\circ} \mathrm{C}$. The water was changed between trials. Each mouse was allowed to swim for a maximum of $6 \mathrm{~min}$, and the immobility time was recorded during the last $5 \mathrm{~min}$ of the trial. After the trial ended, the mouse was carefully dried with a cloth towel and kept under a heating fan for $1 \mathrm{~h}$ before placing back into the home cage.

2.2.4. Barnes Maze Test. The Barnes maze task is a spatial memory task. The maze apparatus (Techman, BMT-100, Chengdu, China) was a round platform $(75 \mathrm{~cm}$ in diameter) with 18 evenly arranged holes $(7 \mathrm{~cm}$ in diameter, Figure 1(a)). During the training, a target box was placed below one of the holes, which was labeled as the target hole. Training and testing were performed in a 500 lux light environment. The platform was cleaned with $75 \%$ ethanol after each use to avoid smelling interference with the next animal.

The day before the training, a mouse was placed in the target hole for $1 \mathrm{~min}$ to get familiar with the environment. For the spatial training, target holes were maintained in the same location relative to the extramaze cues on each trial. The trial was started by placing a mouse in the center of the platform. The mouse was covered with a cylinder. After $10 \mathrm{~s}$, the mouse was allowed to freely explore the platform for 

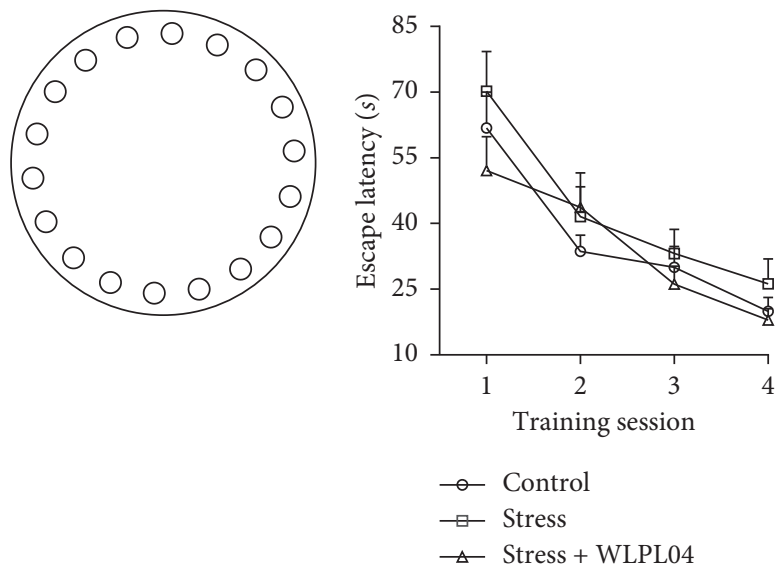

(a)

(b)

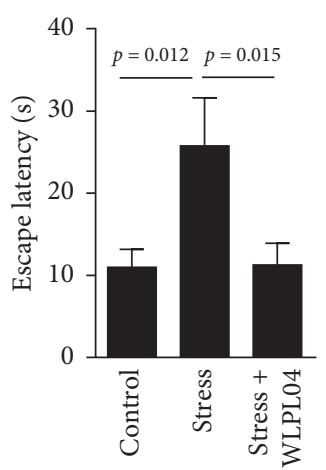

(c)

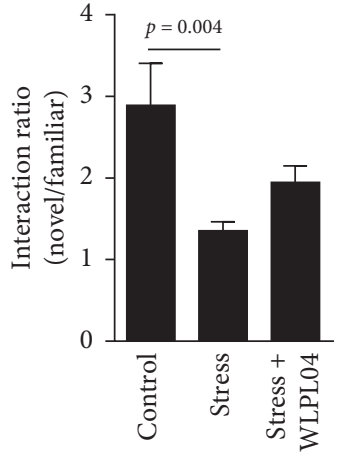

(d)

Figure 1: L. plantarum WLPL04 treatment alleviates the cognitive dysfunctions in chronically stressed mice. (a) Diagram of Barnes maze. (b), (c) Chronic stress impairs spatial memory but not spatial learning in the Barnes maze, and such memory deficit is rescued by the L. plantarum WLPL04 treatment. (d) Chronic stress impairs the novel/familiar object recognition, and such deficit is reversed by the L. plantarum WLPL04 treatment. $n=7$ mice per group.

3 min. If the mouse found the target hole, the mouse was allowed to stay inside the target box for $30 \mathrm{~s}$. If the mouse failed to find the target hole, the mouse was guided to the target box, and the environment light was turned off simultaneously. The mouse was permitted to stay in the box for $30 \mathrm{~s}$. Each mouse was trained for three 3 trials each day for four consecutive days.

For the spatial memory testing, the target box was removed and each mouse underwent a probe trial on the fifth day. The mouse was placed on the center of the platform and allowed to freely explore the platform for $90 \mathrm{~s}$. The latency to find the target box was recorded.

2.2.5. Novel Object Recognition Test. The novel object recognition task was performed in a square arena $(30 \mathrm{~cm} \times 30 \mathrm{~cm} \times 45 \mathrm{~cm})$. Each mouse was placed in the arena to explore for $1 \mathrm{~min}$ to get familiar with the environment. One day later, two identical objects were placed in two distinct corners of the arena, and the mouse was allowed to explore the arena for $10 \mathrm{~min}$. On the next day, one of the two identical objects (familiar objects) was replaced by a novel object (nonfamiliar object). The novel object was different in shape and color with the familiar ones. The mouse was placed in the arena to freely explore for $10 \mathrm{~min}$. The exploration behavior of the mouse was video recorded for analysis. The arena was cleaned with $75 \%$ ethanol after each use.

\subsection{DNA Extraction and High-Throughput DNA Sequencing.} After the chronic restraint stress, the mouse feces was collected from every cage and immediately placed in $1.5 \mathrm{~mL}$ screw-capped tubes for DNA extraction. Prior to the DNA extraction, each sample tube was added with sterilized phosphate-buffered saline (PBS) solution $(6 \mathrm{ml}, 0.05 \mathrm{M}, \mathrm{pH}$ 7.4), shaken for 5-10 min, and centrifuged for $5 \mathrm{~min}$ at $500 \mathrm{rpm}$. The deposits were collected in Eppendorf tubes, and such processes were repeated thrice. The deposits were

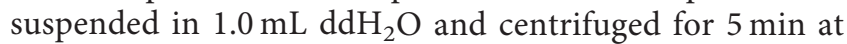
$14000 \mathrm{rpm}$. The resulting deposits were dissolved in $200 \mu \mathrm{l}$ absolute ethanol (precooled at $-20^{\circ} \mathrm{C}$ ) and centrifuged for $2 \mathrm{~min}$ at $14,000 \mathrm{rpm}$. The supernatant was discarded, and the process was repeated thrice. The total DNA was extracted from the feces by using the TIANamp Bacteria DNA Kit (TIANGEN, DP302, Beijing, China) in accordance with the manufacturer's instructions. The extracted genomic DNA was sent to Personal, Inc. (Personal Bio Inc., 
Shanghai, China) for high-throughput sequencing and analysis.

The V3/4 region of the 16S rRNA gene was amplified using universal primers 338F ( $5^{\prime}$-ACTCCTACGGGAGGCAGCAG-3') and 806R ( $5^{\prime}$-GGACTACHVGGGTWTCTAAT $\left.-3^{\prime}\right)$. The PCR product was extracted from $2 \%$ agarose gels, purified, and quantified. The sequencing was carried out using the Illumina MiSeq platform and $2 \times 300 \mathrm{bp}$ reagent kit for paired-end sequencing (GenBank accession number PRJNA673977). Operational taxonomic units (OTUs) were clustered with $97 \%$ similarity cutoff, and chimeric sequences were identified and removed using the QIIME analysis tools (Quantitative Insights into Microbial Ecology, v1.8.0; http://qiime.org/).

2.4. Enzyme-Linked Immunosorbent Assay (ELISA). The truck blood was collected and centrifuged at $3400 \mathrm{rpm}$ for $20 \mathrm{~min}$ to measure the 5-HT level. The supernatant was collected and stored at $-80^{\circ} \mathrm{C}$ for further analysis. The $5-\mathrm{HT}$ level was measured using the commercial ELISA kit (ab133053, Abcam, Cambridge, UK). In brief, varying concentrations of standard and sample solutions were added into the ELISA plates. All ELISA measurements were performed in two replicates.

2.5. Protein Extraction and Western Blot. Brain tissues were quickly removed, washed with PBS, and homogenized with phenylmethanesulfonyl fluoride (Sigma-Aldrich, 78830, Wisconsin, USA). The total protein concentration was measured using the BCA protein assay kit (Thermo Scientific, 23235, New York, USA). Protein extracts were used for Western blot to quantify the levels of brain-derived neurotrophic factor (BDNF), tropomyosin-related kinase B (TrkB), and glyceraldehyde-3-phosphate dehydrogenase (GAPDH). After the measurement and the adjustment of protein concentration, samples were added with $4 \mathrm{x}$ loading buffer, heated at $100^{\circ} \mathrm{C}$ for $10 \mathrm{~min}$, and loaded onto the SDS-PAGE. Proteins were transferred onto polyvinylidene difluoride membranes (Merck Millipore, ISEQ00010 and IPVH00010, Massachusetts, USA) for $2 \mathrm{~h}$ at $56 \mathrm{~V}$ in the transfer buffer. Membranes were blocked with 5\% nonfat milk in Tris-buffered saline (TBST) for $2 \mathrm{~h}$ at room temperature on an orbital shaker. The membranes were then cut into several parts in accordance with the goal protein molecular weight and incubated with primary antibodies (antiBDNF antibody, $1: 1000$, Abcam, ab108319, Cambridge, UK; anti-TrkB antibody, 1:5000, Abcam, ab187041, Cambridge, UK; and anti-GAPDH antibody, 1:1000, BioRad, MCA4739, California, USA) overnight at $4^{\circ} \mathrm{C}$. The membranes were washed thrice with TBST ( 5 min each time) and incubated with horseradish peroxidase- (HRP-) conjugated IgG secondary antibodies (goat anti-mouse IgG HRP, 1: 3000, Cw0102s, Beijing, China; and goat anti-rabbit IgG HRP, $1: 3000$, Cw0103s, Beijing, China) for $2 \mathrm{~h}$ at room temperature. Signals were visualized using the Gel DocTM EZ System (BioRad, California, USA), and the relative levels of BDNF, TrkB, and GAPDH proteins were analyzed using the "Image J" Software (https://imagej.nih.gov/ij/).
2.6. Statistical Analysis. Statistical analysis was performed using the GraphPad Prism 6. Continuous variables were presented as the mean \pm standard error of the mean. Results were analyzed using unpaired one-way and two-way ANOVA.

The Chaol index was determined as follows:

$$
S_{\text {Chaol }}=S_{\text {obs }}+\frac{n 1(n 1-1)}{2(n 2+1)}
$$

where $S_{\text {Chaol }}$ is the estimated number of OTUs (operational taxonomic units), $S_{\text {obs }}$ is the observed number of OUTs, $n 1$ is the number of OTU that have one sequence, and $n 2$ is the number of OTU that have two sequences.

The Shannon index was commonly calculated as follows:

$$
H_{\text {Shannon }}=-\sum_{i=1}^{R} p_{i} \ln p_{i}
$$

where $p_{i}$ is the proportion of characters belonging to the $i^{\text {th }}$ type of letter in the string of interest, and $R$ denotes the actual number of types.

\section{Results and Discussion}

3.1. L. plantarum WLPL04 Rescues Stress-Induced Anxiety/ Depressive-Like Behaviors. The behavioral assessment with the open-field test showed that the chronically stressed mice spent significantly less time in the central area of the open field compared with the control mice, indicating that the chronically stressed mice exhibited the anxiety phenotype. This phenotype was not observed in the stress + WLPL04 group of mice (Figures 2(a) and 2(b), control: $22.22 \pm 2.159 \mathrm{~s}$; stress: $12.41 \pm 1.115 \mathrm{~s}$; stress + WLPL04:17.73 $\pm 1.798 \mathrm{~s}$; and one-way ANOVA, $F(2,27)=7.928, p=0.0020)$.

The elevated plus maze test showed that the L. plantarum WLPL04 treatment could rescue the chronic stress-induced anxiety phenotype. The stressed mice spent significantly less time (control: $43.93 \pm 7.197 \mathrm{~s}$; stress: $23.61 \pm 3.078 \mathrm{~s}$; stress + WLPL04: $34.13 \pm 2.552 \mathrm{~s} ;$ and one-way ANOVA, $F$ $(2,27)=4.571, p=0.0195)$ and executed significantly less entries (control: 6.300 \pm 1.146 ; stress: $3.300 \pm 0.3000$; stress + WLPL04: $6.900 \pm 1.286$; and one-way ANOVA, $F(2$, $27)=3.651, p=0.0395)$ in the open arms of the maze compared with the control mice. This anxiety-like phenotype was not observed in the stress + WLPL04 group of mice (Figures 2(c) and 2(d)).

The forced swimming test was performed to examine the effect of the L. plantarum WLPL04 treatment on the depressive-like behavior. The stressed mice had significantly more immobility time (control: $37.17 \pm 5.846 \mathrm{~s}$; stress: $71.43 \pm 8.680 \mathrm{~s} ;$ stress + WLPL04 : $40.57 \pm 5.467 \mathrm{~s}$; and oneway ANOVA, $F(2,32)=7.670, p=0.0019)$ compared with the control mice, indicating that the chronically stressed mice exhibited the depressive-like phenotype. This phenotype was not observed in the stress + WLPL04 group of mice (Figure 2(e)).

Overall, these results suggested that the L. plantarum WLPL04 treatment could alleviate the anxiety and the 

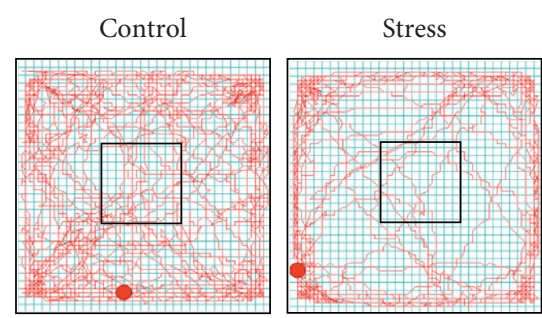

Stress + WLPL04

(a)

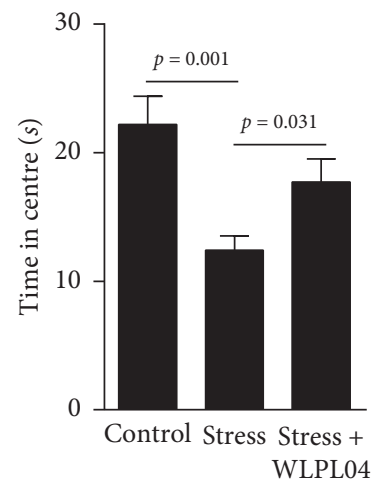

(b)

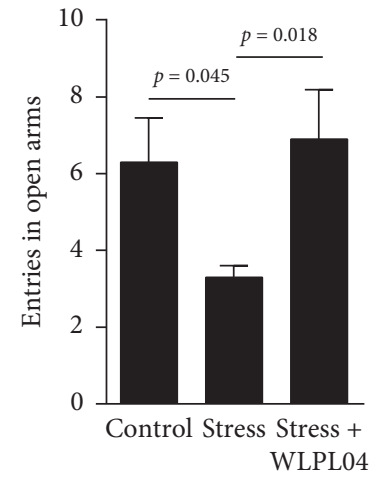

(d)

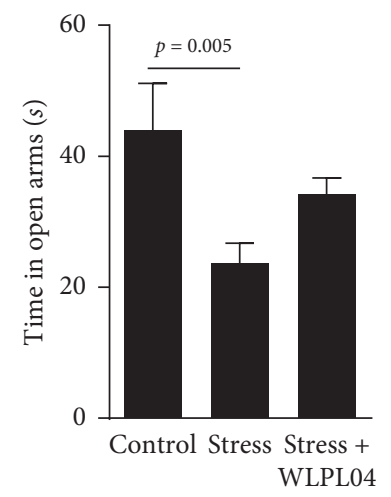

(c)

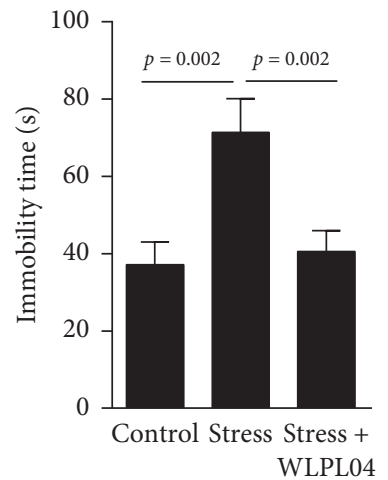

(e)

FIgURE 2: L. plantarum WLPL04 treatment reduces anxiety-like behaviors in chronically stressed mice. (a) The moving trajectory of the three groups of mice in the open-field test. (b) Chronically stressed mice exhibit significantly $(p<0.01)$ less time in the central area of the open field, and such behavioral phenotype is alleviated by the L. plantarum WLPL04 treatment. Chronically stressed mice have spent (c) significantly $(p<0.01)$ less time and (d) significantly $(p<0.05)$ fewer entries into the open arms in the elevated plus maze test, and such behavioral phenotypes are alleviated by the L. plantarum WLPL04 treatment. (e) Chronically stressed mice demonstrate significantly $(p<0.01)$ longer immobility time, and such behavioral phenotype is alleviated by the L. plantarum WLPL04 treatment. $n=7-12$ mice per group.

depressive-like behavioral phenotypes induced by chronic stress.

\subsection{L. plantarum WLPL04 Alleviates Stress-Induced Cognitive} Functional Deficits. The Barnes maze and novel object recognition tests were performed to examine whether the $L$. plantarum WLPL04 treatment could alleviate the cognitive deficits induced by the chronic restraint stress. In the Barnes maze test, which measured the hippocampus and prefrontal cortex-dependent spatial learning and memory, the stressed and the control mice performed equally well during the training, indicating an intact ability of spatial learning (session 1: control: $61.85 \pm 8.202 \mathrm{~s}$; stress: $70.22 \pm 9.081 \mathrm{~s}$; stress + WLPL04:52.10 $\pm 7.771 \mathrm{~s} ; \quad$ session 2: control: $33.64 \pm 3.709 \mathrm{~s} ; \quad$ stress: $41.59 \pm 6.798 \mathrm{~s} ; \quad$ stress + WLPL04 : $43.70 \pm 7.901 \mathrm{~s}$; session 3: control: $30.01 \pm 4.748 \mathrm{~s}$; stress: $33.14 \pm 5.496 \mathrm{~s} ;$ stress + WLPL04 : $26.16 \pm 4.148 \mathrm{~s}$; session 4 : control: $\quad 19.95 \pm 3.223 \mathrm{~s}$; $\quad$ stress: $26.20 \pm 5.703 \mathrm{~s}$; stress + WLPL04:17.94 $\pm 2.660 \mathrm{~s}$; and two-way ANOVA, $F$ $(6,162)=0.6035, p=0.7272)$. However, in the spatial memory in the probe trial, compared with the control mice, the stressed mice spent a significantly longer time (control: $10.96 \pm 2.218 \mathrm{~s} ; \quad$ stress: $25.76 \pm 5.814 \mathrm{~s} ; \quad$ stress + WLPL04 : $11.28 \pm 2.641 \mathrm{~s}$; and one-way ANOVA, $F(2,39)=4.693$, $p=0.0149$ ) to find the target box, indicating impaired spatial 
memory. Such impairment was not observed in the stress + WLPL04 group of mice, suggesting the protective effect of the L. plantarum WLPL04 on the spatial memory consolidation (Figures 1(b) and 1(c)).

In the novel object recognition test, which measures the prefrontal cortex-dependent cognitive function $[23,24]$, the control mice preferred to interact with the novel objects. By contrast, the stressed mice spent equal time interacting with novel and familiar objects, indicating a deficit in the novel object recognition. Such deficit was not observed in the stress + WLPL04 group of mice, suggesting that the $L$. plantarum WLPL04 could protect the cognitive ability for novel object recognition (Figure 1(d), control: $2.889 \pm 0.5159 ; \quad$ stress: $1.354 \pm 0.1111 ; \quad$ stress + WLPL04 : $1.946 \pm 0.2031$; and one-way ANOVA, $F(2,18)=5.620$, $p=0.0127)$.

Overall, these results suggested that the L. plantarum WLPL04 treatment could alleviate the cognitive deficits induced by chronic stress.

\subsection{L. plantarum WLPL04 Reverses the Reduction in the} Intestinal Microbiota Diversity Caused by Stress. The composition of intestinal microbiota was analyzed using the high-throughput DNA sequencing. The analysis of the relative abundance of the detected bacteria within each sample at the phylum level revealed a decrease in the abundance of Firmicutes and Actinobacteria and an increase in the abundance of Bacteroidetes in the stressed mice compared with those in the control mice (Figure 3(a)). Such changes were not observed in the stress + WLPL04 group of mice (Figure 3(a)). The Chaol and the Shannon analyses showed that the species richness (Chaol) and the microbiota diversity (Shannon) in the feces were reduced by the chronic restraint stress, and such reductions were somehow reversed upon probiotic treatment with the L. plantarum WLPL04 (Figures 3(b) and 3(c), Chao1: control: 930.0 4 49.00; stress: $662.0 \pm 11.00 ; \quad$ stress + WLPL04:772.3 $\pm 21.36 ; \quad$ one-way ANOVA, $F(2,4)=18.70, p=0.0093$; Shannon: control: $6.700 \pm 0.2900 ;$ stress: $5.325 \pm 0.08500 ;$ stress + WLPL04 : $5.990 \pm 0.3262$; and one-way ANOVA, $F(2,4)=4.606$, $p=0.0917)$. Thus, the chronic stress reduced the intestinal microbiota diversity, and such reduction could be reversed by the L. plantarum WLPL04 treatment.

\subsection{L. plantarum WLPL04 Reverses the Decrease in the Serum} 5-HT Level Caused by Stress. The 5-HT, a key element in the gut-brain axis, acts as a neurotransmitter in the central and the enteric nervous systems. The 5 -HT plays an important role in learning, memory, and emotion regulation in the central nervous systems. The analysis of the serum 5-HT level revealed a significant decrease in the 5-HT level in stressed mice compared with that in the control mice. This decrease was not observed in the stress + WLPL04 group of mice (Figure 4, control: $130.6 \pm 9.672 \mathrm{ng} / \mathrm{mL}$; stress: $90.78 \pm 8.945 \mathrm{ng} / \mathrm{mL}$; stress + WLPL0 $: 122.7 \pm 12.65 \mathrm{ng} / \mathrm{mL}$; and one-way ANOVA, $F(2,6)=4.004, p=0.0786)$, suggesting that the L. plantarum WLPL04 could produce a protective effect on the chronically stressed mice by maintaining the serum 5-HT at a normal level.

3.5. L. plantarum WLPL04 Prevents the Stress-Induced Decrease in BDNF and its Receptor TrkB. The BDNF, a member of neurotrophic factors, plays an important role in the nervous system development and learning/memory function [25]. Previous reports show that early-life events regulate the expression of neurotrophic factors [26]. Here, the expression levels of BDNF and its receptor TrkB in the $\mathrm{mPFC}$ of mice were measured using the Western blot. As shown in Figure 5, the chronic stress significantly reduced the levels of $\mathrm{BDNF}$ and the $\operatorname{TrKB}$ protein, and these reductions were not detected in the stress + WLPL04 group of mice (BDNF: control: $\quad 100.0 \pm 2.699 \%$; $\quad$ stress: $\quad 65.57 \pm 7.401 \%$; stress + WLPL04:99.68 $\pm 9.028 \%$; one-way ANOVA, F (2, $6)=8.182, p=0.0193$; TrkB : control: $100.0 \pm 7.444 \%$; stress: $50.64 \pm 2.565 \%$; sStress + WLPL04 : $82.82 \pm 7.694 \%$; and oneway ANOVA, $F(2,6)=15.54, p=0.0042)$. These results suggested that the L. plantarum WLPL04 could prevent the stress-induced decrease in BDNF and its receptor TrkB.

\section{Discussion}

The present study shows that the chronic restraint stress induces anxiety/depressive-like behaviors and results in cognitive deficits, causes an abnormal change in the intestinal microbiota, and reduces the levels of 5-HT, BDNF, and TrkB. These changes can be alleviated with the L. plantarum WLPL04 treatment, providing an experimental basis for the therapeutic application of $L$. plantarum on anxiety/depression and cognitive dysfunctions.

A growing body of studies suggests that the probiotic treatment can reverse the stress-induced intestinal dysbiosis and behavioral abnormality. The beneficial effects of probiotics include promoting host digestion, supporting the immune system, and managing the intestinal microbiota $[27,28]$. Lactobacillus and Bifidobacteria, as the representative bacteria of Firmicutes and Actinobacteria, respectively, are reported to reduce anxiety symptoms in patients with chronic fatigue syndrome [29]. A probiotic formulation combining L. acidophilus Rosell-52 and Bifidobacterium longum Rosell-175 exerts a beneficial effect on the gastrointestinal symptoms in individuals affected by chronic stress [30]. The oral administration of B. longum 1714 or Bifidobacterium breve 1205 for six weeks reduces anxiety-like behavioral phenotypes in anxious BALB/c mice [31]. Several factors increase the risk of depression, including stress and environment and gut microbiota [32, 33]. Our study shows that four-week chronic restraint stress could cause depression. Previous studies show that gut microbiota can modulate depressive-like behavior. Mice treated with a combination of Lactobacillus helveticus R0052 and Bifidobacterium longum R0175 present improvements in depression-like behavior [34]. CCFM1025 treatment significantly reduced anxiety and depression-like behaviors induced by chronic unpredictable mild stress [35]. Mice received $1 \times 10^{9} \mathrm{cfu} \cdot L$. rhamnosus daily for four weeks could 


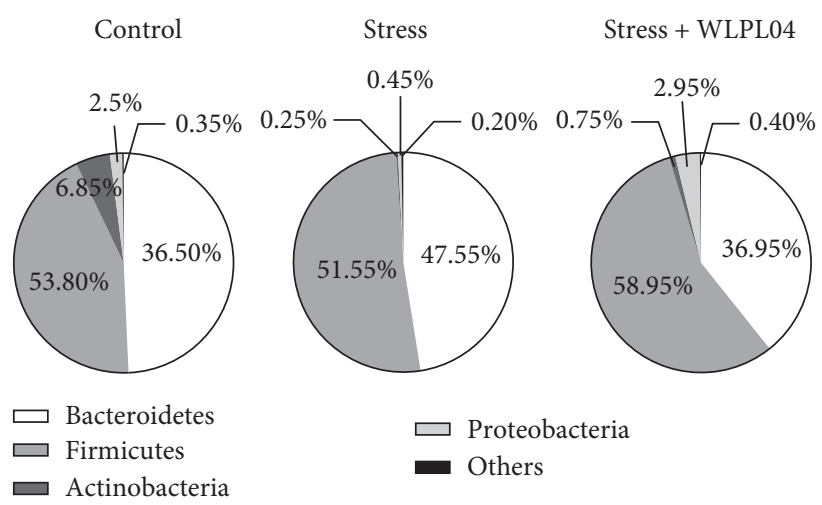

(a)

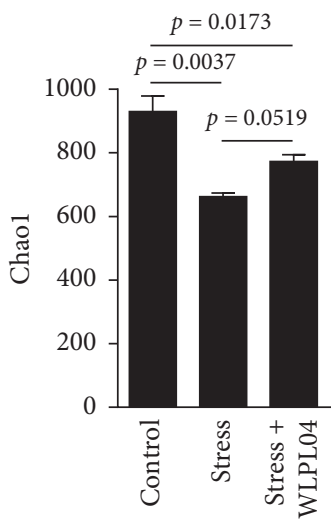

(b)

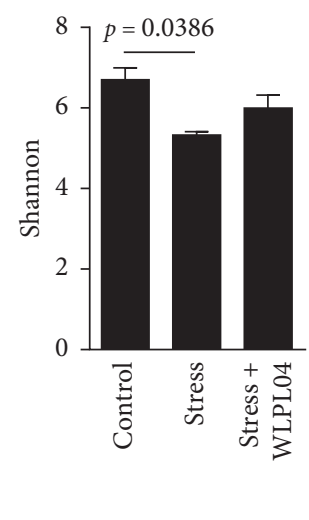

(c)

Figure 3: L. plantarum WLPL04 treatment rescues the negative change in the intestinal microbiota. (a) Aggregate microbiota composition at the phylum level in the fecal samples of experimental mice. Chronic stress significantly reduces the number of Firmicutes, and such negative effect is reversed by the L. plantarum WLPL04 treatment. Bacterial diversity and species richness as indicated by the (b) Chaol and the (c) Shannon indices. The chronic stress tends to destroy the diversity and destroy the species richness, and such negative effects are rescued by the L. plantarum WLPL04 treatment. $n=12$ mice per group.

alleviate anxiety and depression-related behaviors [36]. Data from the open-field test and elevated plus maze test in our study also show that L. plantarum WLPL04 alleviated anxiety and depressive-like behaviors induced by chronic restraint stress, suggesting its antianxiety and antidepression effect of L. plantarum WLPL04.

The link between microbiome composition and neurodevelopment has been proposed for a long time [4]. Microbiota has been reported to influence the neurodevelopment. The alterations of the gut microbiota may affect the neurodevelopment and could be mediated by microbiota via microbiota-gut-brain axis [37, 38]. Neurodevelopment-related molecular, such as BDNF, has been found to be related with the microbiota-gut-brain axis, and they are susceptible to modulations $[4,39]$. The microbiotagut-brain axis is reported to regulate neuropsychiatric diseases [40]. Alterations of gut microbiota could influence strongly on the neurodevelopment. The L. helveticus NS8 treatment improves cognitive deficit and anxiety-like behaviors in hyperammonemia rats. The two-month administration of $L$. plantarum MTCC1325 ameliorates the cognitive deficits in Alzheimer's disease [16]. The long-term treatment of Lactobacillus paracasei K71 may alleviate the age-dependent cognitive decline in mice [41]. The dysbiosis and behavioral deficits caused by prenatal stress can be prevented by treating the dam and the offspring mice with Lactobacillus-containing probiotics and indigenous Lactobacillus reuteri, respectively $[42,43]$. In the present study, the L. plantarum WLPL04 treatment rescues cognitive deficits in chronically stressed mice, providing evidence that this probiotic treatment can benefit the host by alleviating stressinduced cognitive disorders.

The stress exposure is known to significantly change the gastric acid secretion, gastrointestinal motility, and mucous levels, which can influence the ability of microbes to colonize within the gastrointestinal tract. The stress can alter the composition of intestinal microbiota. For instance, Bailey et al. have reported that a social stressor reduces the relative abundance of Bacteroidetes in mice [5]. The change in the microbiota diversity can be detected as early as $2 \mathrm{~h}$ after stress exposure [44]. A study in nonhuman primates indicates that stress during pregnancy affects the infant gut microbiota by reducing Bifidobacteria and Lactobacilli [45]. The present study shows a consistent result, indicating that the chronic restraint stress induces changes in bacterial species and diversity. 


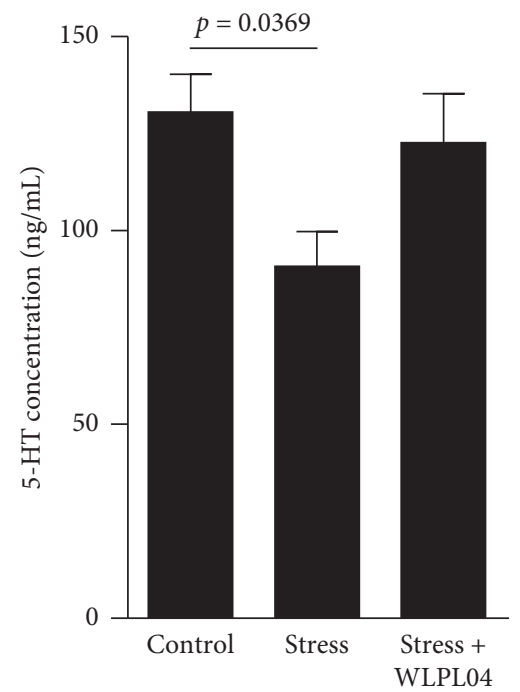

FIgURE 4: Chronic stress causes a decrease in the serum 5-HT concentration, and such effect is reversed by the L. plantarum WLPL04 treatment. Three independent experiments were conducted in each sample group.

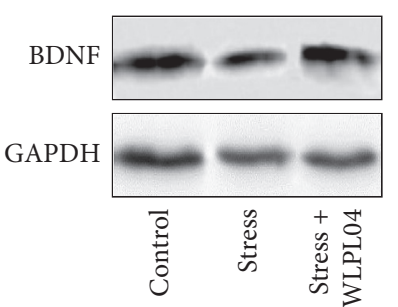

(a)

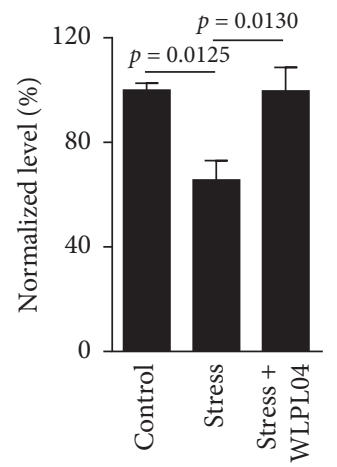

(c)

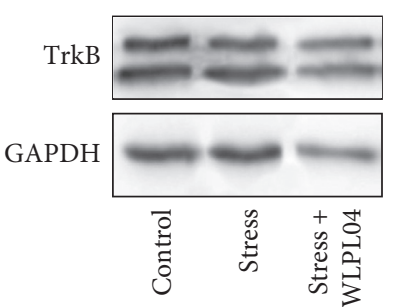

(b)

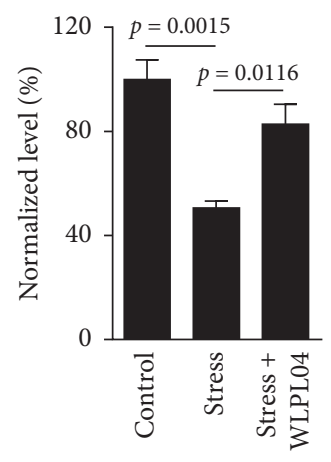

(d)

Figure 5: Protein levels of brain-derived neurotrophic factor (BDNF) and tropomyosin receptor kinase $B$ (TrkB) in the mPFC of mice. Chronic stress reduces the expressions of (a), (c) BDNF and (b), (d) TrkB, and such effects are rescued by the L. plantarum WLPL04 treatment. Three independent experiments were conducted in each sample group.

Although research shows the validity of probiotics, many scholars remain cautious. Suez et al. have found that the potential postantibiotic probiotic benefits may be offset by a compromised gut mucosal recovery [46]. Zmora has confirmed that the empiric probiotic supplementation may be limited and persistently affect the gut mucosa, meriting the development of new personalized probiotic approaches [47]. The effects of probiotic may be dependent on the strain. A probiotic formulation exhibits beneficial effects for stressed animals but has no benefit or may cause harm to normal ones [48]. Lactic acid bacteria are often considered to promote health but are reported to be associated with bad outcomes, including susceptible to inescapable electric stress [49], increased severity of psychotic dysfunctions [50], and high levels of proinflammatory [51]. Lactic acid bacteria are also reported to be relatively abundant in persons with schizophrenia and bipolar disorders $[52,53]$. The discrepancy may be explained by differences in study design and 
methodologies. In the present study, the L. plantarum WLPL04 is supplied as drinking supplement for chronically stressed mice, and results provide further evidence that this probiotic treatment can rescue the intestinal microbiota composition and support the hypothesis that the L. plantarum WLPL04 can benefit the host by alleviating stressinduced mood disorders. The 5 -HT is produced in the brain and the gastrointestinal tract and can be detected in the blood. Gershon and Tack have reported that the gastrointestinal tract contains most of the body's 5-HT [54]. The synthesis and the metabolism of 5-HT in the brain and periphery are believed to be independent. To our knowledge, $90 \%$ of the 5 -HT is produced by enterochromaffin cell and stored in granule cells [55]. When stimulus factors are involved, the 5-HT stored in granule cells is released into the blood [56]. The 5-HT produced in the brain cannot pass the blood-brain barrier and cannot enter blood.

The microbiota is involved in regulating the host's 5-HT level. Germ-free mice have significantly low serum serotonin [57]. Approximately 50\% of the gut-derived 5-HT is regulated by the gut microbiota. Spore-forming bacteria, which are dominated by the Clostridiaceae and the Turicibacteraceae [57], produce short-chained fatty acids and tryptamine, which influence TPH-1 expression, 5-HT synthesis, and/or 5-HT release [58, 59]. The long-term diet supplementation with L. paracasei K71 elevates the serum serotonin level [41]. Consistent with those of the previous studies, our results show that the L. plantarum WLPL04 can reverse the serum 5-HT level of chronically restrained mice. The L. plantarum WLPL04 may affect the 5-HT synthesis in the gut and rescue the body's 5-HT concentration to a physiological level in the stressed mice.

The serotoninergic system plays an important role in the mood regulation [60]. The 5-HT has long been recognized as a key contributor to the regulation of mood and anxiety and is strongly associated with the etiology of major depression [61]. Early studies demonstrate that disabling the serotonergic system completely either by preventing the 5-HT production or by lesioning the 5-HT neurons leads to widespread behavioral consequences ranging from cognitive deficits to avoidance behaviors [52-65]. Increasing the bioavailability of the 5-HT in serotoninergic synapses effectively alleviates depressive symptoms [66]. In the present study, the serum 5-HT concentration is significantly decreased in the chronically stressed mice, and such decrease is alleviated by the L. plantarum WLPL04 treatment.

As the most abundant neurotrophic factor, the BDNF affects several aspects of brain functions. The expression of BDNF is dependent on the activity and regulated by internal and environmental factors. Increasing studies have shown that the stress downregulates the expression of BDNF [67-69]. The intestinal microbiota regulates the level of BDNF in the central nervous system [70]. For example, the administration of antimicrobials transiently alters the composition of microbiota and reduces the expression of BDNF in the hippocampus of mice [69]. Moreover, the hippocampal BDNF expression is upregulated after the mice are orally administered with the probiotic B. longum 1714 [31]. Consistently, the present study has found that the chronic restraint stress reduces the expression of BDNF and its receptor TrkB in the $\mathrm{mPFC}$ and impairs mPFC-dependent cognitive functions, and such effects are alleviated by the supplementation of the L. plantarum WLPL04.

\section{Conclusions}

In summary, the L. plantarum WLPL04 treatment can alleviate anxiety/depressive-like behaviors, the abnormal change in intestinal microbiota, and the reduced levels of 5HT, BDNF, and TrkB induced by chronic stress, providing an experimental basis for its therapeutic application on anxiety/depressive mood disorders.

\section{Data Availability}

The data used to support the findings of this study are available from the corresponding author upon request.

\section{Conflicts of Interest}

The authors declare that there are no conflicts of interest.

\section{Acknowledgments}

This study was supported by the Natural Science Foundation of Jiangxi Province (Grant nos. 2016BAB215206, 20171ACB20002, and 20171BAB204019).

\section{References}

[1] C. Heim and C. B. Nemeroff, "The impact of early adverse experiences on brain systems involved in the pathophysiology of anxiety and affective disorders," Biological Psychiatry, vol. 46, no. 11, pp. 1509-1522, 1999.

[2] M. Bonati and A. Clavenna, "The epidemiology of psychotropic drug use in children and adolescents," International Review of Psychiatry, vol. 17, no. 3, pp. 181-188, 2005.

[3] S. H. Rhee, C. Pothoulakis, and E. A. Mayer, "Principles and clinical implications of the brain-gut-enteric microbiota axis," Nature Reviews Gastroenterology \& Hepatology, vol. 6, no. 5, pp. 306-314, 2009.

[4] S. M. Collins, M. Surette, and P. Bercik, "The interplay between the intestinal microbiota and the brain," Nature Reviews Microbiology, vol. 10, no. 11, pp. 735-742, 2012.

[5] M. T. Bailey, S. E. Dowd, J. D. Galley, A. R. Hufnagle, R. G. Allen, and M. Lyte, "Exposure to a social stressor alters the structure of the intestinal microbiota: implications for stressor-induced immunomodulation," Brain, Behavior, and Immunity, vol. 25, no. 3, pp. 397-407, 2011.

[6] P. Bercik, E. F. Verdu, J. A. Foster et al., "Chronic gastrointestinal inflammation induces anxiety-like behavior and alters central nervous system biochemistry in mice," Gastroenterology, vol. 139, no. 6, pp. 2102-2112, 2010.

[7] M. G. Gareau, E. Wine, D. M. Rodrigues et al., "Bacterial infection causes stress-induced memory dysfunction in mice," Gut, vol. 60, no. 3, pp. 307-317, 2011.

[8] T. G. Dinan, C. Stanton, and J. F. Cryan, "Psychobiotics: a novel class of psychotropic," Biological Psychiatry, vol. 74, no. 10, pp. 720-726, 2013.

[9] F. Shanahan, T. G. Dinan, P. Ross, and C. Hill, "Probiotics in transition," Clinical Gastroenterology and Hepatology, vol. 10, no. 11, pp. 1220-1224, 2012. 
[10] A. C. Logan and M. Katzman, "Major depressive disorder: probiotics may be an adjuvant therapy," Medical Hypotheses, vol. 64, no. 3, pp. 533-538, 2005.

[11] H. Eutamene and L. Bueno, "Role of probiotics in correcting abnormalities of colonic flora induced by stress," Gut, vol. 56, no. 11, pp. 1495-1497, 2007.

[12] M. Bernardeau, J. P. Vernoux, S. Henridubernet et al., "Safety assessment of dairy microorganisms: the lactobacillus genus," International Journal of Food Microbiology, vol. 126, no. 3, pp. 271-273, 2008.

[13] M. I. Masood, M. I. Qadir, J. H. Shirazi, and I. U. Khan, "Beneficial effects of lactic acid bacteria on human beings," Critical Reviews in Microbiology, vol. 37, no. 1, p. 91, 2011.

[14] X. Peng, J. Meng, T. Chi et al., "Lactobacillus plantarum ndc 75017 alleviates the learning and memory ability in aging rats by reducing mitochondrial dysfunction," Experimental and Therapeutic Medicine, vol. 8, no. 6, pp. 1841-1846, 2014.

[15] N. Mallikarjuna, K. Praveen, and K. Yellamma, "Role of Lactobacillus plantarum MTCC1325 in membrane-bound transport ATPases system in alzheimer's disease-induced rat brain,” Bioimpacts, vol. 6, no. 4, pp. 203-209, 2016.

[16] M. Nimgampalle and Y. Kuna, "Anti-alzheimer properties of probiotic, lactobacillus plantarum mtcc 1325 in alzheimer's disease induced albino rats," Journal of Clinical and Diagnostic, vol. 11, no. 8, pp. KC01-KC05, 2017.

[17] R. Arroyo, V. Martín, A. Maldonado, E. Jiménez, L. Fernández, and J. M. Rodríguez, "Treatment of infectious mastitis during lactation: antibiotics versus oral administration of lactobacilli isolated from breast milk," Clinical Infectious Diseases, vol. 50, no. 12, pp. 1551-1558, 2010.

[18] M. P. Díaz-Ropero, R. Martín, S. Sierra et al., “Two Lactobacillus strains, isolated from breast milk, differently modulate the immune response," Journal of Applied Microbiology, vol. 102, no. 2, pp. 337-343, 2010.

[19] J. Maldonado, F. Lara-Villoslada, S. Sierra et al., "Safety and tolerance of the human milk probiotic strain Lactobacillus salivarius CECT5713 in 6-month-old children," Nutrition, vol. 26, no. 11-12, pp. 1082-1087, 2010.

[20] M. Jiang, F. Zhang, C. Wan et al., "Evaluation of probiotic properties of Lactobacillus plantarum WLPL04 isolated from human breast milk," Journal of Dairy Science, vol. 99, no. 3, pp. 1736-1746, 2016.

[21] B. Liu, L. Zhao, C. Yue, M. Qian, and M. Xie, "Changes in gonadal function at different stages of chronic restraint stressinduced depression animals," Physiology \& Behavior, vol. 210, Article ID 112656, 2019.

[22] Y. J. Yang, Z. Y. Cheng, H. Tang et al., "Neonatal maternal separation impairs prefrontal cortical myelination and cognitive functions in rats through activation of wnt signaling," Cerebral Cortex, vol. 27, pp. 2871-2884, 2016.

[23] A. Arias-Cavieres, T. Adasme, G. Sánchez et al., “Aging impairs hippocampal-dependent recognition memory and LTP and prevents the associated RyR up-regulation," Frontiers in Aging Neuroscience, vol. 9, p. 111, 2017.

[24] M. R. Leite, M. H. Marcondes Sari, M. L. De Freitas et al., "Caffeine and diphenyl diselenide improve long-term memory impaired in middle-aged rats," Experimental Gerontology, vol. 53, pp. 67-73, 2015.

[25] K. Yamada and T. Nabeshima, "Brain-derived neurotrophic factor/TrkB signaling in memory processes," Journal of Pharmacological Sciences, vol. 91, no. 4, pp. 267-270, 2003.

[26] N. Sudo, Y. Chida, Y. Aiba et al., "Postnatal microbial colonization programs the hypothalamic-pituitary-adrenal system for stress response in mice," The Journal of Physiology, vol. 558, no. 1, pp. 263-275, 2004.

[27] C. Hill, F. Guarner, G. Reid et al., "The international scientific association for probiotics and prebiotics consensus statement on the scope and appropriate use of the term probiotic," Nature Reviews Gastroenterology \& Hepatology, vol. 11, no. 8, pp. 506-514, 2014.

[28] G. T. Rijkers, W. M. De Vos, R.-J. Brummer, L. Morelli, G. Corthier, and P. Marteau, "Health benefits and health claims of probiotics: bridging science and marketing," British Journal of Nutrition, vol. 106, no. 9, pp. 1291-1296, 2011.

[29] A. V. Rao, A. C. Bested, T. M. Beaulne et al., "A randomized, double-blind, placebo-controlled pilot study of a probiotic in emotional symptoms of chronic fatigue syndrome," Gut Pathogens, vol. 1, no. 1, p. 6, 2009.

[30] L. Diop, S. Guillou, and H. Durand, "Probiotic food supplement reduces stress-induced gastrointestinal symptoms in volunteers: a double-blind, placebo-controlled, randomized trial," Nutrition Research, vol. 28, no. 1, pp. 1-5, 2008.

[31] H. M. Savignac, B. Kiely, T. G. Dinan, and J. F. Cryan, "Bifidobacteriaexert strain-specific effects on stress-related behavior and physiology in BALB/c mice," Neurogastroenterology \& Motility, vol. 26, no. 11, pp. 1615-1627, 2014.

[32] M. Berk, L. J. Williams, F. N. Jacka et al., "So depression is an inflammatory disease, but where does the inflammation come from?” BMC Medicine, vol. 11, p. 200, 2013.

[33] M. Jokela, M. Virtanen, G. D. Batty, and M. Kivimäki, "Inflammation and specific symptoms of depression," JAMA Psychiatry, vol. 73, no. 1, pp. 87-88, 2016.

[34] A. Ait-Belgnaoui, A. Colom, V. Braniste et al., "Probiotic gut effect prevents the chronic psychological stress-induced brain activity abnormality in mice," Neurogastroenterology \& Motility, vol. 26, no. 4, pp. 510-520, 2014.

[35] P. Tian, K. J. O’Riordan, Y. K. Lee et al., “Towards a psychobiotic therapy for depression: bifidobacterium breve ccfm1025 reverses chronic stress-induced depressive symptoms and gut microbial abnormalities in mice," Neurobiology of Stress, vol. 12, 2020.

[36] R. Janik, L. A. M. Thomason, A. M. Stanisz, P. Forsythe, J. Bienenstock, and G. J. Stanisz, "Magnetic resonance spectroscopy reveals oral Lactobacillus promotion of increases in brain GABA, N-acetyl aspartate and glutamate," Neuroimage, vol. 125, pp. 988-995, 2016.

[37] G. B. Rogers, D. J. Keating, R. L. Young, M.-L. Wong, J. Licinio, and S. Wesselingh, "From gut dysbiosis to altered brain function and mental illness: mechanisms and pathways," Molecular Psychiatry, vol. 21, no. 6, pp. 738-748, 2016.

[38] K. Rea, T. G. Dinan, and J. F. Cryan, "The microbiome: a key regulator of stress and neuroinflammation," Neurobiology of Stress, vol. 4, pp. 23-33, 2016.

[39] H. Park and M.-M. Poo, "Neurotrophin regulation of neural circuit development and function," Nature Reviews Neuroscience, vol. 14, no. 1, pp. 7-23, 2013.

[40] T. G. Dinan and J. F. Cryan, "Brain-gut-microbiota axis and mental health," Psychosomatic Medicine, vol. 79, no. 8, pp. 920-926, 2017.

[41] H. Corpuz, S. Ichikawa, M. Arimura et al., "Long-term diet supplementation with lactobacillus paracasei K71 prevents age-related cognitive decline in senescence-accelerated mouse prone 8," Nutrients, vol. 10, no. 6, p. 762, 2018.

[42] S. Leclercq, F. M. Mian, A. M. Stanisz et al., "Low-dose penicillin in early life induces long-term changes in murine 
gut microbiota, brain cytokines and behavior," Nature Communications, vol. 8, Article ID 15062, 2017.

[43] S. A. Buffington, G. V. Di Prisco, T. A. Auchtung, N. J. Ajami, J. F. Petrosino, and M. Costa-Mattioli, "Microbial reconstitution reverses maternal diet-induced social and synaptic deficits in offspring," Cell, vol. 165, no. 7, pp. 1762-1775, 2016.

[44] J. D. Galley, M. C. Nelson, Z. Yu et al., "Exposure to a social stressor disrupts the community structure of the colonic mucosa-associated microbiota," BMC Microbiology, vol. 14, no. 1, p. 189, 2014.

[45] T. B. Michael, R. L. Gabriele, and L. C. Christopher, "Prenatal stress alters bacterial colonization of the gut in infant monkeys," Journal of Pediatric Gastroenterology and Nutrition, vol. 38, no. 4, pp. 414-421, 2004.

[46] J. Suez, N. Zmora, G. Zilberman-Schapira et al., "Post-antibiotic gut mucosal microbiome reconstitution is impaired by probiotics and improved by autologous FMT," Cell, vol. 174, no. 6, p. 1406, 2018.

[47] N. Zmora, G. Zilberman-Schapira, J. Suez et al., "Personalized gut mucosal colonization resistance to empiric probiotics is associated with unique host and microbiome features," Cell, vol. 174, no. 6, pp. 1388-1405, 2018.

[48] J. Barouei, M. Moussavi, and D. M. Hodgson, "Effect of maternal probiotic intervention on HPA axis, immunity and gut microbiota in a rat model of irritable bowel syndrome," PLoS One, vol. 7, no. 10, Article ID e46051, 2012.

[49] K. Zhang, Y. Fujita, L. J. Chang et al., “Abnormal composition of gut microbiota is associated with resilience versus susceptibility to inescapable electric stress," Translational Psychiatry, vol. 9, p. 231, 2019.

[50] Y. He, T. Kosciolek, J. Tang et al., "Gut microbiome and magnetic resonance spectroscopy study of subjects at ultrahigh risk for psychosis may support the membrane hypothesis," European Psychiatry, vol. 53, pp. 37-45, 2018.

[51] A. Painold, S. Mörkl, K. Kashofer et al., "A step ahead: exploring the gut microbiota in inpatients with bipolar disorder during a depressive episode," Bipolar Disorders, vol. 21, no. 1, pp. 40-49, 2019.

[52] E. Castro-Nallar, M. L. Bendall, M. Pérez-Losada et al., "Composition, taxonomy and functional diversity of the oropharynx microbiome in individuals with schizophrenia and controls," PeerJ, vol. 3, no. 8, p. e1140, 2015.

[53] M. Berk, F. Kapczinski, A. C. Andreazza et al., "Pathways underlying neuroprogression in bipolar disorder: focus on inflammation, oxidative stress and neurotrophic factors," Neuroscience \& Biobehavioral Reviews, vol. 35, no. 3, pp. 804-817, 2011.

[54] M. D. Gershon and J. Tack, "The serotonin signaling system: from basic understanding to drug development for functional GI disorders," Gastroenterology, vol. 132, no. 1, pp. 397-414, 2007.

[55] V. Erspamer and B. Asero, "Identification of enteramine, the specific hormone of the enterochromaffin cell system, as 5hydroxytryptamine," Nature, vol. 169, no. 4306, pp. 800-801, 1952.

[56] C. C. Toh, "Release of 5-hydroxytryptamine (serotonin) from the dog's gastro-intestinal tract," The Journal of Physiology, vol. 126, no. 2, pp. 248-254, 1954.

[57] J. M. Yano, K. Yu, G. P. Donaldson et al., "Indigenous bacteria from the gut microbiota regulate host serotonin biosynthesis," Cell, vol. 161, no. 2, pp. 264-276, 2015.

[58] S. N. Spohn and G. M. Mawe, "Non-conventional features of peripheral serotonin signalling - the gut and beyond," Nature
Reviews Gastroenterology \& Hepatology, vol. 14, no. 7, pp. 412-420, 2017.

[59] J. M. Blander, R. S. Longman, I. D. Iliev, G. F. Sonnenberg, and D. Artis, "Regulation of inflammation by microbiota interactions with the host," Nature Immunology, vol. 18, no. 8, pp. 851-860, 2017.

[60] Y. Liu, J. Zhao, and W. Guo, "Emotional roles of monoaminergic neurotransmitters in major depressive disorder and anxiety disorders," Frontiers in Psychology, vol. 9, p. 2201, 2018.

[61] M. J. Robson, M. A. Quinlan, and R. D. Blakely, "Immune system Activation and depression: roles of serotonin in the central nervous system and periphery," ACS Chemical Neuroscience, vol. 8, no. 5, pp. 932-942, 2017.

[62] C. Mazer, J. Muneyyirci, K. Taheny et al., "Serotonin depletion during synaptogenesis leads to decreased synaptic density and learning deficits in the adult rat: a possible model of neurodevelopmental disorders with cognitive deficits," Brain Research, vol. 760, no. 1, pp. 68-73, 1997.

[63] I. González-Burgos, A. R. Del Angel-Meza, G. Barajas-López, and A. Feria-Velasco, "Tryptophan restriction causes longterm plastic changes in corticofrontal pyramidal neurons," International Journal of Developmental Neuroscience, vol. 14, no. 5, pp. 673-679, 1996.

[64] G. R. Breese, R. A. Vogel, and R. A. Mueller, "Biochemical and behavioral alterations in developing rats treated with 5,7dihydroxytryptamine," The Journal of Pharmacology and Experimental Therapeutics, vol. 205, no. 3, pp. 587-595, 1978.

[65] P. M. Whitaker-Azmitia, X. Zhang, and C. Clarke, "Effects of gestational exposure to monoamine oxidase inhibitors in rats: preliminary behavioral and neurochemical studies," Neuropsychopharmacology, vol. 11, no. 2, pp. 125-132, 1994.

[66] M. J. Owens and C. B. Nemeroff, "Role of serotonin in the pathophysiology of depression: focus on the serotonin transporter," Clinical Chemistry, vol. 40, no. 2, pp. 288-295, 1994.

[67] J. M. Pizarro, L. A. Lumley, W. Medina et al., "Acute social defeat reduces neurotrophin expression in brain cortical and subcortical areas in mice," Brain Research, vol. 1025, no. 1, pp. 10-20, 2004.

[68] F. Karege, G. Vaudan, M. Schwald et al., "Neurotrophin levels in postmortem brains of suicide victims and the effects of antemortem diagnosis and psychotropic drugs," Molecular Brain Research, vol. 136, no. 1, pp. 29-37, 2005.

[69] N. Gervasoni, J.-M. Aubry, G. Bondolfi et al., "Partial normalization of serum brain-derived neurotrophic factor in remitted patients after a major depressive episode," Neuropsychobiology, vol. 51, no. 4, pp. 234-238, 2005.

[70] P. Bercik, E. Denou, J. Collins et al., "The intestinal microbiota affect central levels of brain-derived neurotropic factor and behavior in mice," Gastroenterology, vol. 141, no. 2, pp. 599-609, 2011. 